\title{
Soft Structures of Monoids and Fields
}

\author{
P. Gnanachandra, A. Muneesh kumar
}

\begin{abstract}
The ultimate purpose of this article is to introduce and examine some new kind of algebraic structures such as Soft topological fields, Soft topological groups and Soft monoids with illustrating counter examples. Also we established that every Soft field over a topological field is a Soft topological field and we have given an example GF(16), the finite field of 16 elements which is a soft topological field
\end{abstract}

Keywords: Soft topological field, Soft topological groups, Soft monoids, Soft field

AMS Classification: 06D72, 22A05, 28C10, $54 H 11$

\section{INTRODUCTION}

In our day-to-day life, we look out problems with unreliabilities. To handle the lack of unreliability and to solve the problems related to uncertainty, a short time ago numberless theories have been developed like Rough sets [Pawlak 1982], Fuzzy sets [Zadeh, 1965], Vague sets [Gau and Buehrer, 1993]. However, these methodologies have their own risks. To circumvent these difficulties, Molodtstev [1999] developed Soft set theory to deal with unreliability. The development of Soft set theory is whistle stop now-adays. Contrivance of Soft theory in algebraic structures was instigated by Aktas and Cagman [2]. They deliberated Soft groups and come into possession of some of their fundamental traits. Acar.et.at [1] initiated the notion of Soft rings, which is a framework of subrings of a ring over a ring. In the milieu, we define the notation of Soft monoids and soft topological fields as an extension work carried out by Nazmul and Samanta [16].

\section{NOTATIONS AND PRELIMINARIES}

We call the following definitions with illustrative examples for the outpouring of this article.

Definition 2.1. Let $\mu$ be an initial universal set and B be a set of parameters. Let $A \subseteq B$. The pair $(\eta, A)$ is a soft set over $\mu$, here $\eta$ is a mapping given by $\eta: A \rightarrow P(\mu)$. Put another way, a soft set over $\mu$ is a parameterized family of subsets of the universal set $\mu$. For $\mathrm{e} \in \mu, \eta(\mathrm{e})$ represents the set of e-appropriate elements of $(\eta, A)$.

Considering the definitions on Soft sets, we refer to [11], [14], [15]. Notwithstanding, we anamnesis a part of definitions and results on algebra and soft sets.

Example 2.2. Suppose $\mu=$ Set of all real numbers on the closed interval $[\mathrm{a}, \mathrm{b}], \mathrm{B}=$ Set of parameters. Each parameter is a word or a sentence. (i.e.) $B=\{$ Open, Closed, Compact, Connected $\}$. In this case, to define a soft set means to point out closed set, connected set and so on.

Revised Manuscript Received on March 17, 2020.

*Correspondence Author: P.Gnanachandra

P.Gnanachandra*, Centre for Research and Post Graduate Studies in Mathematics, Ayya Nadar Janaki Ammal College, Sivakasi, India. Email: pgchandra07@gmail.com

A.Muneesh kumar, Centre for Research and Post Graduate Studies in Mathematics, Ayya Nadar Janaki Ammal College, Sivakasi, India. Email:muneeshkumarar@gmail.com

(C) The Authors. Published by Blue Eyes Intelligence Engineering and Sciences Publication (BEIESP). This is an open access article under the CC BY-NC-ND license (http://creativecommons.org/licenses/by-nc-nd/4.0/)
We consider below the same in detail, where $e_{1}$ stands for the parameter 'compact', $e_{2}$ stands for the parameter 'closed', $\mathrm{e}_{3}$ stands for the parameter 'connected', and $\mathrm{e}_{4}$ stands for the parameter 'open'. Suppose that $\eta\left(e_{1}\right)=\{A \subseteq$ [a,b] : Every open cover for $A$ has finite subcover $\}, \eta\left(\mathrm{e}_{2}\right)=$ $\{[\alpha, \beta] \subseteq[\mathrm{a}, \mathrm{b}]: \alpha, \beta \in \mathbb{R}\}, \eta\left(\mathrm{e}_{3}\right)=\{\mathrm{A} \subseteq[\mathrm{a}, \mathrm{b}]:$ Separation does not exists for $\mathrm{A}$ in $[\mathrm{a}, \mathrm{b}]\}, \eta\left(\mathrm{e}_{4}\right)=\{(\alpha, \beta) \subseteq[\mathrm{a}, \mathrm{b}]: \alpha, \beta$ $\in \mathbb{R}\}$. The soft set $(\eta, A)$ is a parameterized family of the subsets of the set $\mu$. $\eta\left(e_{1}\right)$ means set of all subsets of $\mu$ which are compact whose functional values is the set $\{\mathrm{A} \subseteq$ [a,b] : Every open cover for $\mathrm{A}$ in $[\mathrm{a}, \mathrm{b}]$ has a finite subcover . Hence the soft set $(\eta, A)$ is the collection of approximations given below : (compact, $\{\mathrm{A} \subseteq[\mathrm{a}, \mathrm{b}]$ : Every open cover for $\mathrm{A}$ in $[\mathrm{a}, \mathrm{b}]$ has a finite subcover $\}$ ), (closed, $\{[\alpha, \beta] \subseteq[\mathrm{a}, \mathrm{b}]: \alpha, \beta \in \mathbb{R}\}$ ), (connected, $\{\mathrm{A} \subseteq[\mathrm{a}, \mathrm{b}]$ : Separation does not exists for $A$ in $[a, b]\})$, (open, $\{(\alpha, \beta) \subseteq$ $[\mathrm{a}, \mathrm{b}]: \alpha, \beta \in \mathbb{R}\})=(\eta, \mathrm{A})$.

Definition 2.3. A topological group is a group $G$ which is also a topological space such that the multiplication map of $G \times G$ into $G$ by $(x, y) \rightarrow$ xy (where $x, y \in G$ ) and the inverse map of $\mathrm{G}$ onto $\mathrm{G}$ by $\mathrm{x} \rightarrow \mathrm{x}^{-1}$ are continuous.

The unit circle $S^{1}$, the matrix group $\operatorname{GL}(n, \mathbb{R})$ and the direct product of $S^{1}$ with the product topology are some examples of topological groups.

\section{A. Soft Topological Groups And Soft Topological Rings}

In this section, we recollect the definitions by Tariq Shah et.at. [17] and proved examples in our own way.

Definition 2.4. Let $G$ be a group and let $\tau$ be a topology defined on $G$. Let $(\eta, A)$ be a non-null soft set defined over $G$. Then $(\eta, A, \tau)$ is called a soft topological group over $G$ if

i. $\quad \eta(a)$ is a subgroup of $G$ for all $a \in A$

The mapping $(\mathrm{x}, \mathrm{y}) \rightarrow x y^{-1}$ of $\eta(a) \times \eta(a)$ onto $\eta(a)$ is continuous for all $\mathrm{a} \in \mathrm{A}$

Example 2.5. Consider the group $G=S_{3}$ and let $\mathrm{A}=$ $\left\{e_{1}, e_{2}, e_{3}\right\}$ be the set of parameters and the base for the topology $\tau$

is $\mathcal{B}=\{\{e\},\{(12)\},\{23\},\{(13)\},\{(123)\},\{(132)\}\}$. Define $\eta\left(e_{1}\right)=\{e\}, \eta\left(e_{2}\right)=\{e,(12)\}, \eta\left(e_{3}\right)=\{e,(123),(132)\}$. Here $\eta\left(e_{1}\right)$ is a trivial identity subgroup of G. Since $\eta\left(e_{2}\right)$ is generated by $\{(12)\}$ and $\eta\left(e_{3}\right)$ is generated by $\{(123)\}$, so $\eta\left(e_{2}\right)$ and $\eta\left(e_{3}\right)$ are also subgroups of $\mathrm{G}$. Here $\eta\left(e_{i}\right) \times$ $\eta\left(e_{i}\right) \rightarrow \eta\left(e_{i}\right), i=1,2,3$ is continuous. Hence $(\eta, A, \tau)$ is a soft topological group.

Example 2.6. Consider $G=\mathbb{Z}$, the infinite cyclic group. Let $\mathrm{A}=\left\{e_{1}, e_{2}, e_{3}, \ldots\right\}$ and $\tau=\{\varnothing, \mathbb{Z}, 2 \mathbb{Z}, 4 \mathbb{Z}, 8 \mathbb{Z}, 16 \mathbb{Z}, \ldots$. Define the set valued functions by $\eta\left(e_{1}\right)=2 \mathbb{Z}, \eta\left(e_{2}\right)=$ $4 \mathbb{Z}, \eta\left(e_{3}\right)=8 \mathbb{Z}, \eta\left(e_{4}\right)=16 \mathbb{Z}, \ldots$ (i.e) $\eta\left(e_{n}\right)=2^{n} \mathbb{Z}$. Clearly $\eta\left(e_{n}\right)$ is a subgroup of $\mathrm{G}$ for all $n \in \mathbb{N}$. Also the maps $(x, y) \rightarrow x y^{-1}$ of $\eta(a) \times \eta(a)$ onto $\eta(a)$ is continuous, for all $a \in A$. For example, consider $\eta\left(e_{1}\right) \times \eta\left(e_{1}\right)$ onto $\eta\left(e_{1}\right)$. The function is continuous at $(2,8)$. For, Consider the neighbourhood $2 \mathbb{Z}$ of -6 ,

Published By:

Blue Eyes Intelligence Engineering DOI: 10.35940/ijeat.C5767.049420

Journal Website: www.ijeat.org

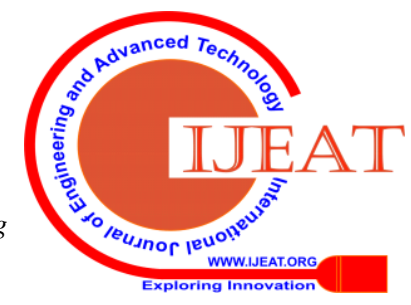




\section{Soft Structures of Monoids and Fields}

there exist neighbourhoods $2 \mathbb{Z}$ and $4 \mathbb{Z}$ of 2 and 8 such that $2 \mathbb{Z}-4 \mathbb{Z}=\{ \pm 2, \pm 6, \ldots\} \subseteq 2 \mathbb{Z}$. Similarly we can check the continuity of other points. Hence $(\eta, A, \tau)$ is a soft topological group.

Definition 2.7. Let $\tau$ be a topology defined on a ring R. Let $(\eta, A)$ be a non-null soft set defined over $\mathrm{R}$. Then the triplet $(\eta, A, \tau)$ is called a Soft topological ring over $\mathrm{R}$ if

i. $\quad \eta(a)$ is a subring of $\mathrm{R}$ for all $a \in A$

ii. $(x, y) \rightarrow x+y$ of $\eta(a) \times \eta(a)$ onto $\eta(a)$ is continuous for all $a \in A$

iii. $(x, y) \rightarrow x . y$ of $\eta(a) \times \eta(a)$ onto $\eta(a)$ is continuous for all $a \in A$

Example 2.8. Consider $R=\mathbb{Z}_{4}=\{0,1,2,3\}$ and let $\mathrm{A}=$ $\{2,3\}$. Consider the topology $\tau=\left\{\varnothing, \mathbb{Z}_{4},\{0\},\{0,2\},\{0,1\}\right.$, $\{0,1,2\}\}$. Define the set valued function $\eta: A \rightarrow \mathcal{P}\left(\mathbb{Z}_{4}\right)$ by $\eta(a)=\{b \in R: a \cdot b=0\}$. Now, $\eta(2)=\{b \in R: 2 b=0\}=$ $\{0,2\}$ and $\eta(3)=\{b \in R: 3 b=0\}=\{0\}$. Both are subrings of R. Consider the map $f:\{0,2\} \times\{0,2\} \rightarrow\{0,2\}$ such that $(0,0) \rightarrow 0,(0,2) \rightarrow 2,(2,0) \rightarrow 2,(2,2) \rightarrow 0$. Now, clearly $g:\{0\} \times\{0\} \rightarrow\{0\}$ is a zero map. Now we check the continuity of $f$ at $(2,0)$. Take any arbitrary neighbourhood of 2 (the image of $(2,0)$ ) say $\{0,2\}$. Then there exist $\{0,2\}$ of 2 and $\{0\}$ of 0 such that $\{0,2\}+\{0\} \subseteq\{0,2\}$. Also take another arbitrary neighbourhood of 2 say $\{0,1,2\}$. Then there exist $\{0,2\}$ of 2 and $\{0\}$ of 0 such that $\{0,2\}+\{0\} \subseteq\{0,1,2\}$. Similarly we can check the continuity of other points. Hence $f$ and $g$ are continuous. Thus $(\eta, A, \tau)$ is a soft topological ring.

Example 2.9. Consider $R=\mathbb{Z}_{2} \times \mathbb{Z}_{3}=\{(0,0),(0,1)$, (0,2), $(1,0),(1,1),(1,2)\}$ and let $A=\{(2,0),(3,0)\}$. Let $\tau=\left\{\varnothing, \mathbb{Z}_{2} \times\right.$ $\mathbb{Z}_{3},\{(0,0)\},\{(0,1),(1,0)\}, \quad\{(0,0),(0,1),(0,2) \quad\},\{(0,0)$, $(0,1),(0,2),(1,0)\}\}$. Define the set valued functions by $\eta(a)=\{b \in R: a \cdot b=0\}$. Now, $\eta((2,0))=\{(0,0),(1,0)\}$ and $\eta((3,0))=\{(0,0),(0,1),(0,2)\}$. Here both are subrings of R. Now we check the continuity of $f$ from $\eta((2,0)) \times$ $\eta((2,0))$ onto $\eta((2,0))$ and $g$ from $\eta((3,0)) \times \eta((3,0))$ onto $\eta((3,0))$. Consider the point $((1,0),(0,0))$ in the domain of $f$. Take any arbitrary neighbourhood of $(1,0)$ (the image of $((1,0),(0,0)))$ say $\{(0,0),(0,1),(0,2),(1,0)\}$. Then there exist $\{(0,0),(1,0)\}$ of $(1,0)$ and $\{(0,0),(1,0)\}$ of $(0,0)$ such that $\{(0,0),(1,0)\}+\{(0,0),(1,0)\}=\{(0,0),(1,0)\} \subseteq\{(0,0),(1,0)\}$. Similarly we can check the continuity of other points. Hence $(\eta, A, \tau)$ is a soft topological ring.

\section{SOFT MONOIDS}

Throughout the article $\mathrm{M}$ denotes the semigroup with an identity element $e$.

Definition 3.1. Soft Monoid over a monoid $M$ is a non-null soft set $(\eta, A)$ with the property that $\eta(x)$ is a submonoid of $\mathrm{M}$ for all $x \in \operatorname{supp}(\eta, A)$ where $\eta: A \rightarrow \mathcal{P}(M)$ defined by $\eta(x)=\{y \in M:(x, y) \in \rho\}$ where $\rho$ is a relation between A and $\mathrm{M}$.

Example 3.2. Consider the monoid $\mathrm{M}=(Z,$.$) . Let (\eta, A)$ be a soft set over $\mathbb{Z}$ where $\mathrm{A}=\mathbb{N}=\{1,2,3, \ldots\}$ and $\eta: A \rightarrow \mathcal{P}(\mathbb{Z})$ defined by $\eta(x)=\{y \in \mathbb{Z}: x \rho y \leftrightarrow x / y\} \cup\{1\}$. Here

$$
\begin{aligned}
& \eta(1)=\{y \in \mathbb{Z}: 1 / y\} \cup\{1\}=\mathbb{Z} \\
& \eta(2)=\{y \in\{y \in \mathbb{Z}: 2 / y\} \cup\{1\} \\
& =\{0,2,-2,4,-4, \ldots\} \cup\{1\}=2 Z \cup\{1\} \\
& \eta(3)=\{y \in\{y \in \mathbb{Z}: 3 / y\} \cup\{1\} \\
& =\{0,3,-3,6,-6, \ldots\} \cup\{1\}=3 Z \cup\{1\}
\end{aligned}
$$

and so on. In general,

$$
\eta(i)=\{y \in\{y \in \mathbb{Z}: i / y\} \cup\{1\}=i Z \cup\{1\}
$$

Note that $\eta(i)$ is a submonoid of $\mathbb{Z}$ for all $i \in A$. Hence $(\eta, A)$ is a soft monoid over $\mathbb{Z}$.

Proposition 3.3. Let $(\alpha, A)$ and $(\beta, B)$ be soft monoids over $\mathrm{M}$. Then the soft set $(\alpha, A) \wedge(\beta, B)$ is a soft monoid over M if it is non-null.

Proof: By the definition of $\wedge$, we have $(\alpha, A) \wedge(\beta, B)=$ $(\gamma, C)$, where $\gamma(x, y)=\alpha(x) \cap \beta(y)$ for all $(x, y) \in C=$ $A \times B$. Assume $\operatorname{supp}(\gamma, C) \neq \emptyset$. Then $\gamma(x, y)=\alpha(x) \cap$ $\beta(y) \neq \emptyset$. Since $\alpha(x)$ and $\beta(y)$ are soft monoids over M ten the intersection is also a monoid namely $\gamma(x, y)$ for all $(x, y) \in \operatorname{supp}(\gamma, C)$.

Definition 3.4. Let $(\alpha, A)$ and $(\beta, B)$ be a soft monoid over $\mathrm{M}$. Then $(\beta, B)$ is called a Soft submonoid over M if $B \subseteq A$ and $\beta(x)$ is a submonoid of $\alpha(x)$, for all $x \in \operatorname{supp}(\beta, B)$.

Example 3.5. Consider the monoid $M=G L\left(2, \mathbb{F}_{2}\right)$ where $G L\left(2, \mathbb{F}_{2}\right)=\left\{\left(\begin{array}{ll}1 & 0 \\ 0 & 1\end{array}\right),\left(\begin{array}{ll}1 & 1 \\ 0 & 1\end{array}\right),\left(\begin{array}{ll}1 & 0 \\ 1 & 1\end{array}\right),\left(\begin{array}{ll}0 & 1 \\ 1 & 0\end{array}\right),\left(\begin{array}{ll}0 & 1 \\ 1 & 1\end{array}\right),\left(\begin{array}{ll}1 & 1 \\ 1 & 0\end{array}\right)\right\}$ where $\mathrm{A}=\mathrm{M}$ and $\eta: A \rightarrow \mathcal{P}(M)$ defined by $\eta(A)=\{B \in$ $G L\left(2, \mathbb{F}_{2}\right): B \rho A \leftrightarrow B=A^{n}$ for some $n \in \mathbb{N}$. Here

$$
\begin{gathered}
\eta\left(\left(\begin{array}{ll}
1 & 0 \\
0 & 1
\end{array}\right)\right)=\left\{\left(\begin{array}{ll}
1 & 0 \\
0 & 1
\end{array}\right)\right\} \\
\eta\left(\left(\begin{array}{ll}
0 & 1 \\
1 & 0
\end{array}\right)\right)=\left\{\left(\begin{array}{ll}
1 & 0 \\
0 & 1
\end{array}\right),\left(\begin{array}{ll}
0 & 1 \\
1 & 0
\end{array}\right)\right\} \\
\eta\left(\left(\begin{array}{ll}
1 & 0 \\
1 & 1
\end{array}\right)\right)=\left\{\left(\begin{array}{ll}
1 & 0 \\
0 & 1
\end{array}\right),\left(\begin{array}{ll}
1 & 0 \\
1 & 1
\end{array}\right)\right\} \\
\eta\left(\left(\begin{array}{ll}
1 & 1 \\
0 & 1
\end{array}\right)\right)=\left\{\left(\begin{array}{ll}
1 & 0 \\
0 & 1
\end{array}\right),\left(\begin{array}{ll}
1 & 1 \\
0 & 1
\end{array}\right)\right\} \\
\eta\left(\left(\begin{array}{ll}
1 & 1 \\
1 & 0
\end{array}\right)\right)=\left\{\left(\begin{array}{ll}
1 & 0 \\
0 & 1
\end{array}\right),\left(\begin{array}{ll}
1 & 1 \\
1 & 0
\end{array}\right),\left(\begin{array}{ll}
0 & 1 \\
1 & 1
\end{array}\right)\right\}
\end{gathered}
$$

Note that $\eta\left(\left(\begin{array}{ll}1 & 1 \\ 1 & 0\end{array}\right)\right)=\eta\left(\left(\begin{array}{ll}0 & 1 \\ 1 & 1\end{array}\right)\right)$ and $\eta\left(\left(\begin{array}{ll}a & b \\ c & d\end{array}\right)\right)$ is a submonoid of $\mathrm{M}$ for all matrices in $\mathrm{M}$.

Proposition 3.6. Let $(\alpha, A)$ and $(\beta, A)$ be two soft monoids over $\mathrm{M}$. Then

i. The Bi-intersection $(\alpha, A) \widetilde{\Pi}(\beta, A)$ is a monoid over $\mathrm{M}$ if it is non-null.

ii. If $\beta(x) \subset \alpha(x)$ for all $x \in A$, then $(\beta, A)$ is a soft submonoid of $(\alpha, A)$.

iii. $\quad(\alpha, A) \widetilde{\Pi}(\beta, A)$ is a soft monoid of both $(\alpha, A)$ and $(\beta, A)$ if it is non-null.

Proof:

i. By definition of $\Pi$, we have $(\alpha, A) \sqcap(\beta, A)=$ $(\gamma, A)$ where $\gamma(x)=\alpha(x) \cap \beta(x) \neq \varnothing \quad$ which implies $\gamma(x)$ is a submonoid of $\mathrm{M}$ for all $x \in$ $\operatorname{supp}(\gamma, A)$

ii. Assume $\beta(x) \subset \alpha(x)$ for all $x \in A$. Since $\alpha(x)$ is a submonoid of $\mathrm{M}, \beta(x)$ is also a submonoid of $\mathrm{M}$ and by our assumption we have $(\beta, A)$ is a soft submonoid of $(\alpha, A)$.

iii. Here $(\alpha, A) \sqcap(\beta, A)=(\gamma, A)$ where $\gamma(x)=$ $\alpha(x) \cap \beta(x)$. Assume $(\gamma, A)$ is non-null. Also $\gamma(x) \subseteq \alpha(x)$ and $\gamma(x) \subseteq \beta(x)$ and so the result is obtained.

Theorem 3.7. Let $\left(\alpha_{i}, A_{i}\right)_{i \in I}$ be a non-empty family of soft monoids over a semiring $\mathrm{M}$. Then

i. $\quad \tilde{\wedge}\left(\alpha_{i}, A_{i}\right)$ is a soft monoid over $\mathrm{M}$ if it is non-null.

ii. $\quad \widetilde{\Pi}\left(\alpha_{i}, A_{i}\right)$ is a softmonoid over $\mathrm{M}$ if it is non-null.

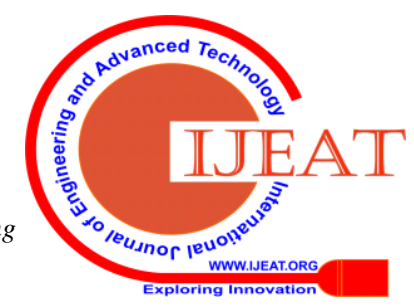


Proof:

i. $\quad$ By the definition of $\wedge, \wedge\left(\alpha_{i}, A_{i}\right)=(\beta, B)$ where $B=\prod A_{i}$ and $\beta(x)=\cap \alpha_{i}$ for all $x=\left(x_{i}\right) \in$ $\operatorname{supp}(\beta, B)$. Assume that $(\beta, B)$ is non-null. Let $x=\left(x_{i}\right) \in \operatorname{supp}(\beta, B)$. Then $\beta(x)=\cap \alpha_{i}(x) \neq \varnothing$. The fact that $\left(\alpha_{i}, A_{i}\right)$ is a soft monoid of $\mathrm{M}$. Hence $\beta(x)$ is a submonoid of $\mathrm{M}$ for all $x=\left(x_{i}\right) \in$ $\operatorname{supp}(\beta, B)$. Thus $\wedge\left(\alpha_{i}, A_{i}\right)$ is a soft monoid over M.

ii. $\quad$ By definition of $\Pi, \Pi\left(\alpha_{i}, A_{i}\right)=(\beta, B)$ where $B=\cap A_{i}$ and $\beta(x)=\cap \alpha_{i}$ for all $x=\left(x_{i}\right) \in$ $\operatorname{supp}(\beta, B)$. Then $\beta(x)=\cap \alpha_{i}(x) \neq \emptyset$. The fact that $\left(\alpha_{i}, A_{i}\right)$ is a soft monoid of $\mathrm{M}$ implies $\alpha_{i}\left(x_{i}\right)$ is a submonoid of M. Hence $\beta(x)$ is a submonoid of M for all $x=\left(x_{i}\right) \in \operatorname{supp}(\beta, B)$. Thus $\wedge\left(\alpha_{i}, A_{i}\right)$ is a soft monoid over $\mathrm{M}$

\section{SOFT TOPOLOGICAL FIELD}

Definition 4.1. Let $\tau$ be a topology defined on a field $\mathrm{F}$ and $(H, A)$ be a non-empty soft set over $\mathrm{F}$. Then the triplet $(H, A, \tau)$ is a Soft Topological Field over $\mathrm{F}$ if for all $a \in A$

i. $\quad H(a)$ is a subfield of $\mathrm{F}$

ii. The mapping $(x, y) \rightarrow x-y$ of $H(a) \times H(a)$ onto $H(a)$ is continuous

iii. The mapping $(x, y) \rightarrow x . y$ of $H(a) \times H(a)$ onto $H(a)$ is continuous.

Example 4.2. Let $A=\mathbb{N}, R=\mathbb{R}$ and $\tau$ be interval topology on $\mathbb{R}$. Let $(H, A)$ be soft set defined by

$$
H=\left\{\begin{array}{c}
\mathbb{Q}(\sqrt{a}) \text { if a is not a perfect square } \\
\mathbb{Q} \text { otherwise }
\end{array}\right.
$$

Here $\mathbb{Q}(\sqrt{a})$ and $\mathbb{Q}$ are the subfields of $\mathbb{R}$, for all $a \in \mathbb{N}$.

Example 4.3. Let $A=\left\{0,1, x^{2}+x, x^{2}+x+1\right\}, F=$ $G F(16)$. Define $\eta(x)$ by $\eta(f(x))=\{g(x): f \sim g \leftrightarrow f . g \in$ A\}.GF(16) $=\left\{a x^{3}+b x^{2}+c x+d+<x^{4}+x+1>\right.$ $: a, b, c, d \in \mathbb{Z} 2 \quad=1,0, x, x 2, x 3, \quad x+1, \quad x 2+1, x 3+1, \quad x+x 2$, $x+x 3, x 2+x 3, x 3+x+1, x 2+x+1, x 3+x 2+x, x 3+x 2+x+1$ Here $\eta(0)=F$ and

$$
\begin{gathered}
\eta(1)=\{g: 1 . g \in A\}=\left\{0,1, x^{2}+x, \quad x^{2}+x+1\right\} \\
\eta\left(x^{2}+x\right)=\left\{0,1, x^{2}+x, x^{2}+x+1\right\} \\
\eta\left(x^{2}+x+1\right)=\left\{0,1, x^{2}+x, x^{2}+x+1\right\}
\end{gathered}
$$

Note that A is a subfield of F. Hence it is a soft topological field.

Theorem 4.4. Every soft field over a topological field is a soft topological field

Proof: Let $(H, \tau)$ be a topological field. Let $\left(H^{\prime}, A\right)$ be a soft fiekd over $\mathrm{F}$. Then by definition $H^{\prime}(a)$ is a subfield of $\mathrm{F}$ for all $a \in A$. Since $\mathrm{H}$ is a topological field the two mappings are continuous for each $H^{\prime}(a)$ and hence $(H, A, \tau)$ is a soft topological field over $(H, \tau)$.

Theorem 4.5. Let $(H, A, \tau)$ and $(G, B, \tau)$ be soft topological field over $F$, where $\tau$ is a topology defined over $F$. Then

i. The Bi-intersection $(H, A, \tau) \widetilde{\Pi}(G, B, \tau)$ is a topological field over $\mathrm{F}$ if it is non-null.

ii. The extended intersection $(H, A, \tau) \cap_{E}(G, B, \tau)$ is a soft topological field over $\mathrm{F}$.

\section{Proof:}

i. By definition, their Bi-intersection over $\mathrm{F}$ is the soft topological set $(K, C, \tau)$ where $C=A \cap B$ and $K(c)=H(c) \cap G(c)$ for all $c \in C$. Since intersection of two subfields of $\mathrm{F}$ is a subfield of $\mathrm{F}$ and since $K(c) \subseteq H(c)$ and $K(c) \subseteq G(c)$ the continuity follows.

ii. By definition, their extended intersection over $\mathrm{F}$ is the soft topological set $(K, C, \tau)$ where $C=A \cup B$

$$
\text { and } K(c)=\left\{\begin{array}{c}
H(c) \text { if } c \in A \backslash B \\
G(c) \text { if } c \in B \backslash A \\
H(c) \cap G(c) \text { if } c \in A \cap B
\end{array}\right.
$$

Here, $K(C)$ is a subfield of $\mathrm{F}$ by (1). If $c \in A \backslash B$ then $K(c)=H(c)$, since the two mappings are continuous for $H(c)$ is also hold for $K(c)$. Similarly if $c \in B \backslash A$ then $K(c)=G(c)$. Since the two mappings are continuous for $G(c)$ it also holds for $K(c)$. If $c \in A \cap B$, then $K(c)=$ $H(c) \cap G(c)$ and so the continuity follows by (1).

Theorem 4.6. If $(H, A, \tau)$ and $(G, B, \tau)$ are two soft topological rings over $\mathrm{F}$. Then $(H, A, \tau) \wedge(G, B, \tau)$ is a soft topological ring over $\mathrm{F}$ if it is non-null.

Proof: Since $(H, A, \tau)$ and $(G, B, \tau)$ are soft topological field over $\mathrm{F}$, by definition $(H, A, \tau) \wedge(G, B, \tau)=\{K, A \times B\}$, where $K((a, b))=H(a) \cap G(b)$ for all $(a, b) \in A \times B$. Since the intersection of two subfields is again a subfield we have $K((a, b))$ is a subfield of $\mathrm{F}$. Since the two mappings are continuous for $H(a)$ and $G(b)$ it also holds for $K((a, b))$.

Definition 4.7. A soft topological field $(H, A, \tau)$ over $\mathrm{F}$ is soft trivial if $H(a)=\{0\}$ for all $a \in A$ and soft whole if $H(a)=F$ for all $a \in A$.

Definition 4.8. Let $(H, A, \tau)$ be a soft topological field over F. Then $(G, B, \tau)$ is said to be a soft topological subfield of $(H, A, \tau)$ if

i. $B \subset A$ and $G(a)$ is a subfield of $H(a)$ for all $a \in \operatorname{supp}(G, B)$

ii. The mapping $(x, y) \rightarrow x-y$ of the topological space $G(a) \times G(a)$ onto $G(a)$ is continuous for all $b \in \operatorname{supp}(G, B)$

iii. For all $b \in B$, the mapping $(x, y) \rightarrow x . y$ of the topological space $G(a) \times G(a)$ onto $G(a)$ is continuous.

Theorem 4.9. If $(H, A, \tau)$ and $(G, B, \tau)$ are two soft topological fields over F, then $K(a) \subset H(a)$ for all $a \in B \subset$ $A$, then $(K, B, \tau)$ is a soft topological subfield $(H, A, \tau)$.

Proof: Since the restriction map of both subtraction and multiplication induced from $H(a) \times H(a)$ is continuous we have $(K, B, \tau)$ is a soft topological subfield of $(H, A, \tau)$.

Example 4.10. In Example 4.3. we take $\mathrm{F}=\mathrm{GF}$ (16) and note that $\eta(x)$ is a subfield of $\mathrm{F}$, for all $x \in A$. Since $\eta(0)=F, \quad \eta(1)=\{g: 1 . g \in A\}=\left\{0,1, x^{2}+x, x^{2}+x+\right.$ 1\}, $\eta\left(x^{2}+x\right)=\left\{0,1, x^{2}+x, x^{2}+x+1\right\}$ and $\eta\left(x^{2}+x+\right.$ 1) $=\left\{0,1, x^{2}+x, x^{2}+x+1\right\}$. Note that the three non-zero elements of this subfield must be cyclic subgroup of order 3 and upto isomorphism there is exactly one such group. Hence this $\eta(x)$ is the only subfield of order 4 .

Published By:

Blue Eyes Intelligence Engineering

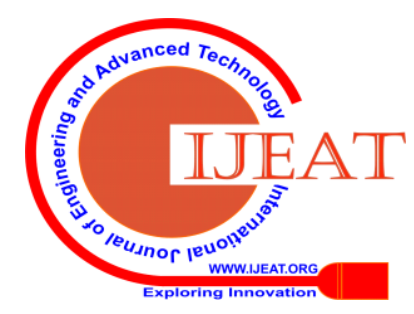




\section{Soft Structures of Monoids and Fields}

\section{CONCLUSION}

In this paper, we investigate some notions such as soft monoids and soft topological fields with illustrated counter examples. Also we prove every soft subfield over a topological field is a soft topological field and we give an example with GF (16), the finite field of 16 elements, which is a soft topological field.

\section{ACKNOWLEDGEMENT}

The Authors express their gramercy to Tariq Shah and Salma Shaheen, Department of Mathematics, Quaid-i-Azam University, Islamabad for their keen engrossment and concernment about Soft topological groups and this article. Also the authors are grateful to the publishing editor of the journal and anonymous reviewers for the worthwhile comments that led to noticeable comments of the article over its original version.

\section{REFERENCES}

1. U. Acar.F.Koyuncu, B.Tanay, Soft sets and soft rings, Computers and Math.with Appl. 59 (2010) 3458 - 3463.

2. H. Aktas, N. Cagman, Soft sets and soft groups, Information Sciences 177 (2007) 2726 - 2735.

3. V.I. Arnsutov, S.T. Glaveatsky, A.V. Mikhalev, Introduction to the theory of topological rings and modules, Marcel Dekker INC, New York, USA, 1996.

4. T. Celik, C. Ekiz and S. Tamak, A new view on soft rings, Hcettepte Journal of Mathematics and Statistics 40 (2) (2011) 273 - 286.

5. T.K. Dutta, S. Kar, K.Das, Power ternary semirings, African Mathematical Union and Springer - Verlsg Heiderberg, 2014.

6. F. Feng, Y.B. Jun, X.Z. Zhao, Soft semirings, Computers and Math. with Appl. 56 (2008) 2621 - 2628.

7. T. Hida, Soft topological group, Annals of Fuzzy Mathematics and Infomatics, Volume 8, No.6, (December 2014), pp. 1001 - 1025.

8. M. Irfan Ali, F.Feng, X.Liu, W.K. Min, M.Shabir, On some new operations in soft set, Computers and Math. with Appl. 57 (2009) $1547-1553$.

9. S. Kar, A. Shikari, Soft Ternary Semirings, Fuzzy Inf.Eng. (2016) 8 $1-15$.

10. D. Molodstov, Soft set theory - first results, Computers and Mathematics with Applications 37 (1999) 19 - 31

11. L.A. Zadeh, Fuzzy sets, Information and Control 8 (1965) 338 - 353.

12. W.L. Gau, D.J. Buehrer, Vague sets, IEEE Transactions on Systems, Man and Cybernetics 23 (2) (1993) 610 - 614.

13. Z. Pawlak, Rough sets, International Journal of Information and Computer Science 11 (1982) 341 - 356.

14. P.K. Maji, R. Biswas, A.R. Roy, On soft set theory, Computers and Mathematics with Applications 45 (2003) 555 - 562.

15. M.I. Ali, M. Shabir, M. Naz, Algebraic structures of soft sets associated with new operations. Computers and Mathematics with Applications 61 (2011) 2647 - 2654.

16. Sk. Nazmul, Sk. Samanta, Soft Topological groups, Kochi Journal of Math 5 (2010) 151 - 161.

17. Tariq Shah, Salma Shaheen, Soft Topological groups and rings, Annals of Fuzzy Mathematics and Infomatics, 7 (5), 725 - 743, (2014).

18. Won Keun Min, A note on soft topological spaces, Computers and Mathematics with Applications, 62 (2011) 3524 - 3528.

\section{AUTHORS PROFILE}

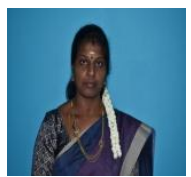

Dr. P. Gnanachandra, completed her Ph.D., in Topology. She has published more than 30 research articles in various reputed journals and Scopus indexed journals.

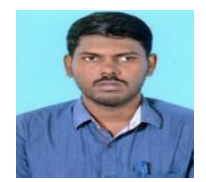

A. Muneesh Kumar doing his Ph.D. in Topological groups. 